\title{
Evaluation of heritability of $\beta$-eudesmol/ hinesol content ratio in Atractylodes lancea De Candolle
}

\author{
Takahiro Tsusaka ${ }^{1,2^{*}}$, Bunsho Makino ${ }^{3}$, Ryo Ohsawa ${ }^{4}$ and Hiroshi Ezura ${ }^{4 *}$
}

\begin{abstract}
Background: Atractylodes lancea De Candolle is a medicinal plant distributed in East Asia. Its rhizome has been used as an important crude drug in traditional Chinese and Japanese medicines for the treatment of numerous diseases and disorders. In recent years, the demand for mass production of the crude drug with a stable quality has increased. Its major active compounds are sesquiterpenoids, such as $\beta$-eudesmol and hinesol that have closely related chemical structures with each other. As the criteria for evaluating the quality of $A$. lancea, the $\beta$-eudesmol/hinesol content ratio is considered important. In $A$. lancea, the ratio could be considered to be influenced by genetic factors, geographical environment factors and these interactions. Few studies of a detail genetic analyses for $\beta$-eudesmol/hinesol content ratio have been reported. Therefore, we evaluated the heritability and genotype-environment interaction on the $\beta$ eudesmol/hinesol content ratio in A. lancea using clonal lines propagated with division of rhizome.

Results: The heritability of the $\beta$-eudesmol/hinesol content ratio in A. lancea was evaluated through the cultivation of clonal lines of $A$. lancea in both different years $(2016,2017)$ and locations (Hokkaido, Ibaraki). Correlations between $\beta$ eudesmol and hinesol contents were identified in all clonal lines, with high correlation coefficients $(r=0.73-0.99)$. The broad-sense heritability of the $\beta$-eudesmol/hinesol content ratio was revealed to be high at 0.92 . The effects of cultivation year were smaller than that of genotype, and few genotype-environment interactions were observed. In addition, the influence of cultivation location was also smaller than that of genotype, and the correlation between the two cultivation locations on the $\beta$-eudesmol/hinesol content ratio was high. The results suggested that the $\beta$ eudesmol/hinesol content ratio in $A$. lancea is highly dependent on genetic factors.

Conclusion: We demonstrate that the heritability of $\beta$-eudesmol/hinesol content ratio is high and that the effects of genetic factors were stronger than that of environmental factors such as cultivation location and year. Our findings suggested that selective breeding and clonal propagation are effective strategies for the production of $A$. lancea with stable qualities for use in the production of crude drugs.
\end{abstract}

Keywords: Atractylodes lancea, $\beta$-Eudesmol, Hinesol, Content ratio, Heritability, Genotype-environment interaction

\footnotetext{
* Correspondence: tsusaka_takahiro@mail.tsumura.co.jp;

ezura.hiroshi.fa@u.tsukuba.ac.jp

${ }^{1}$ Graduate School of Life and Environmental Sciences, University of Tsukuba, Tsukuba, Ibaraki, Japan

${ }^{4}$ Faculty of Life and Environmental Sciences, University of Tsukuba, Ten-nodai

1-1-1, Tsukuba, Ibaraki 305-8572, Japan

Full list of author information is available at the end of the article
}

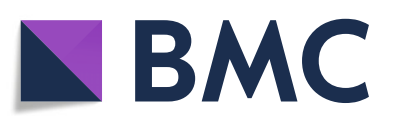

(c) The Author(s). 2020 Open Access This article is licensed under a Creative Commons Attribution 4.0 International License, which permits use, sharing, adaptation, distribution and reproduction in any medium or format, as long as you give appropriate credit to the original author(s) and the source, provide a link to the Creative Commons licence, and indicate if changes were made. The images or other third party material in this article are included in the article's Creative Commons licence, unless indicated otherwise in a credit line to the material. If material is not included in the article's Creative Commons licence and your intended use is not permitted by statutory regulation or exceeds the permitted use, you will need to obtain permission directly from the copyright holder. To view a copy of this licence, visit http://creativecommons.org/licenses/by/4.0/ The Creative Commons Public Domain Dedication waiver (http://creativecommons.org/publicdomain/zero/1.0/) applies to the data made available in this article, unless otherwise stated in a credit line to the data. 


\section{Background}

Atractylodes lancea De Candolle belonging the Compositae family is a perennial medicinal plant widely distributed across East Asia. Its dried rhizome has been used as an important crude drug in traditional Chinese and Japanese medicines [1]. In these medicines, various decoctions containing the crude drug have been used for a treatment of digestive disorders and body fluid imbalance [2, 3]. In modern pharmacological studies, the major active compounds obtained from $A$. lancea rhizomes have been shown to exhibit pharmacological activities on nervous, cardiovascular, and gastrointestinal systems [4]. Additionally, their anticancer, anti-inflammatory, and antimicrobial activities have also been reported [4]. Major active compounds in A. lancea rhizome are sesquiterpenoids such as $\beta$-eudesmol and hinesol, which have closely related chemical structures from each other [5]. Pharmacological activities of these two compounds are resembled, however intensity of pharmacological activity and action mechanism may be different [6] For instance, both of $\beta$-eudesmol and hinesol have mitigation effects against gastric ulcer, but their pharmacological action mechanisms are different [6-8]. Additionally, hinesol has activity to induce apoptosis in human leukemia HL-60 cells, suggesting the possibility that hinesol may be useful anticancer drug, while, it is weak pharmacological activity in $\beta$-eudesmol [9]. In recent years, the demand for mass production of the crude drug with a stable quality has increased [10]. Therefore, keeping the contents of $\beta$ eudesmol and hinesol constant in $A$. lancea rhizomes is important to stabilize its pharmacological activity [11].

In the classical medicine texts, especially in Japan, it has also been mentioned that the crude drug highly suited for medicinal use deposits white cotton-like crystals on a section or epidermis of the dried rhizome [12]. Most of the crystal is comprised of $\beta$-eudesmol and hinesol as major constituents [5]. In addition, the formation of the crystal has been shown to be dependent on not only their high absolute contents but also an equivalent ratio of their contents in the dried rhizome [13]. Consequently, the $\beta$ eudesmol/hinesol content ratio is also assumed as an important quality criteria of $A$. lancea rhizomes as the crude drug suited for medicinal use $[13,14]$.

The quality of $A$. lancea rhizome is closely related to environment of its natural habitats, and $\beta$-eudesmol and hinesol content vary across geographical regions [13, $15]$. We previously demonstrated that $\beta$-eudesmol and hinesol content are strongly influenced by genetic factors [16]. In addition, Takeda et al. [14] suggested that geographical differences in terms of the $\beta$-eudesmol/ hinesol content ratio are mainly caused by genetic differences. Hence, detailed genetic analyses of broad-sense heritability, genotype-environment $(\mathrm{G} \times \mathrm{E})$ interactions, and the effects of environmental factors on the $\beta$ eudesmol/hinesol content ratio are warranted.
In this study, we analyzed data from a previous study [16] on the $\beta$-eudesmol/hinesol content ratio in A. lancea rhizomes and investigated the heritability of this ratio. In particular, a total of 25 clonal lines of A. lancea were grown in an experimental field, and broad-sense heritability of the $\beta$-eudesmol/hinesol content ratio was estimated. Additionally, to investigate stabilities of the traits in annual variability and cultivation locations, we evaluated $\mathrm{G} \times \mathrm{E}$ interactions between genotype and cultivation year or location. Six clonal lines were grown under different years and locations, and two-way ANOVA and correlation analysis between different cultivation years or locations on the $\beta$-eudesmol/hinesol content ratio were performed. In the present study, we attempted to determine the relative effects of genetic factors and environmental factors on the $\beta$-eudesmol/hinesol content ratio in A. lancea.

\section{Results}

Estimation of broad sense heritability on the $\beta$-eudesmol/ hinesol content ratio

In order to evaluate the heritability of the $\beta$-eudesmol/hinesol content ratio in A. lancea, 25 clonal lines were cultivated in an experimental field located in Ibaraki Prefecture (Japan), and the contents of the compounds were determined. Figure 1 shows that the results of correlation analysis between $\beta$-eudesmol and hinesol content in each clonal line. The range of $\beta$-eudesmol contents in all clonal lines were $5.3-34.5 \mathrm{mg} / \mathrm{g}$, and the range of hinesol contents were 4.4-41.1 mg / g. As seen, $\beta$-eudesmol and hinesol content are significantly and positively correlated, with high $r$-values of $0.73-0.99$ in all clonal lines, suggesting $\beta$ eudesmol/hinesol content ratio is stable within a clonal line.

Figure 2 shows variations in the $\beta$-eudesmol/hinesol content ratio across different $A$. lancea clonal lines. The ranges of variation for the $\beta$-eudesmol/hinesol content ratio within an $A$. lacnea clonal line were smaller than varietal differences (Fig. 2). One-way analysis of variance (ANOVA) identified significant differences $(P>0.01)$ in the $\beta$-eudesmol/hinesol content ratio among the $A$. lancea clonal lines (Table 1). From the ANOVA result, the broad-sense heritability of the $\beta$-eudesmol/hinesol content ratio was also revealed to be high at 0.92 (Table 1 ).

\section{Effects of cultivation year on the $\beta$-eudesmol/hinesol content ratio}

To determine the effects of cultivation year on the $\beta$ eudesmol/hinesol content ratio in A. lancea rhizome, 6 clonal lines were grown and analyzed in 2016 and 2017 under the same experimental field located in Ibaraki Prefecture. In two-way ANOVA of the $\beta$-eudesmol/hinesol content ratio, the effects of genotype (G) and cultivation year $(\mathrm{Y})$ were significant, while mean square value for cultivation year was lower than that for genotype (Table 2). No significant differences in $\mathrm{G} \times \mathrm{Y}$ interactions 


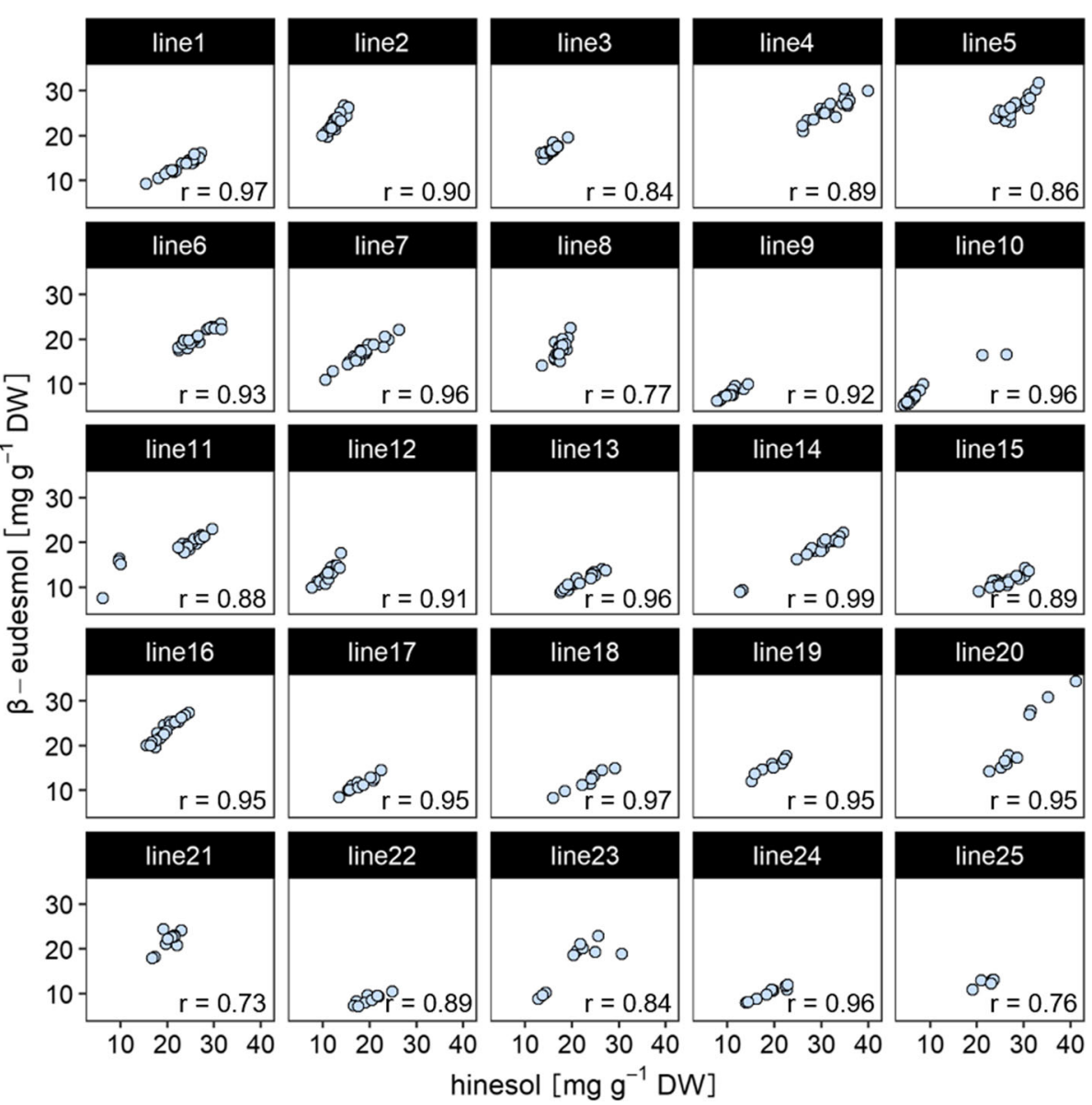

Fig. 1 Correlation between $\beta$-eudesmol and hinesol contents in A. lancea clonal lines. Pearson's correlation coefficients ( $r$ ) were calculated for each clonal line. The number of replicates for each clone is as follows; lines 1-17 $(n=20)$, lines 18-24 $(n=10)$, and line $25(n=5)$

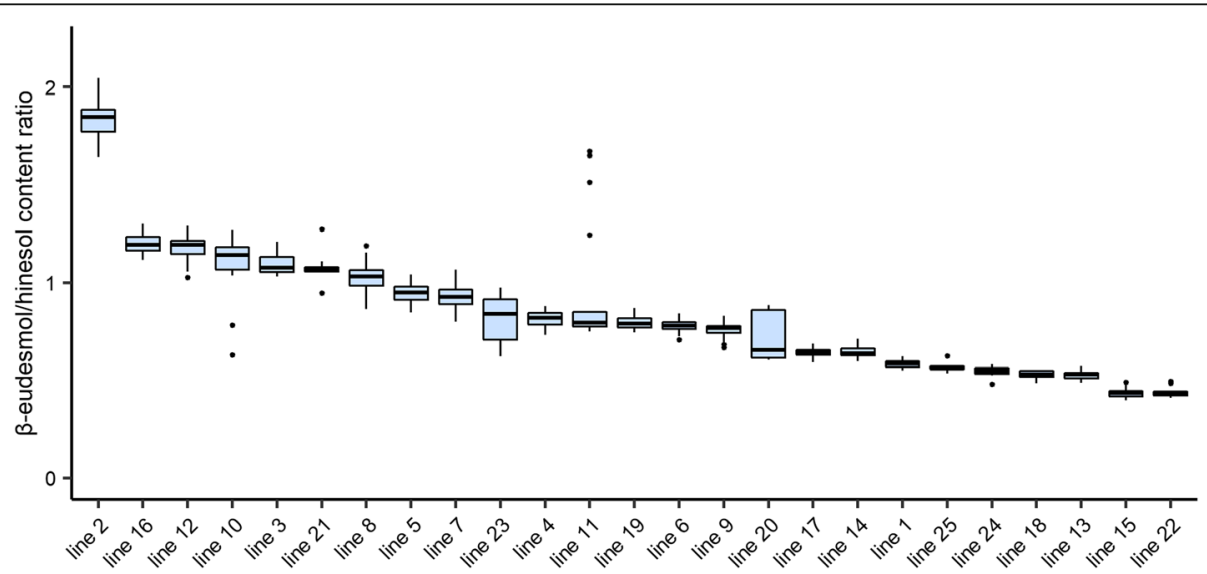

Fig. 2 Range of variations in the $\beta$-eudesmol/hinesol content ratio in A. lancea. Boxes represent 25th-75th percentiles and middle lines represent medians. The vertical lines extend from minimum to maximum values. The numbers of biological replicates for each clonal line is as follows: lines $1-17(n=20)$, lines $18-24(n=10)$, and line $25(n=5)$ 
Table 1 Broad-sense heritability of the $\beta$-eudesmol/hinsol content ratio in A. lancea

\begin{tabular}{llllcccr}
\hline & Df & Mean Sq & $P$-value & Effective replication & Genotypic variance & Environmental variance & Broad-sense heritability \\
\hline Clonal line & 24 & 1.71 & $<2 \mathrm{e}-16^{\mathrm{a}}$ & 16.3 & 0.10 & 0.009 & 0.92 \\
Residuals & 390 & 0.01 & & & & & \\
\hline
\end{tabular}

Df degree of freedom, Mean Sq Mean square; ${ }^{\mathrm{a}} P<0.01$

were identified (Table 2). In addition, the broad-sense heritability of the $\beta$-eudesmol/hinesol content ratio, which was calculated using variance components from two-way ANOVA, was high at 1.00 (Table 2). Few qualitative interactions were observed between genotype and cultivation year (Fig. 3), and the correlation between the two cultivation years on the $\beta$-eudesmol/hinesol content ratio was high $(r=1.00$; Fig. 4$)$.

\section{Effects of cultivation location the $\beta$-eudesmol/hinesol content ratio}

In order to evaluate the effect of cultivation location on the $\beta$-eudesmol/hinesol content ratio, 6 clonal lines were grown at two different locations, Hokkaido and Ibaraki prefecture. Environmental condition of these cultivation locations were differed. Hokkaido is located in northernmost point in Japan, whereas Ibaraki prefecture is located in middle of Japan. The experimental field in Hokkaido was located in $43^{\circ} .01^{\prime} \mathrm{N}, 140^{\circ} .53^{\prime} \mathrm{E}, 8 \mathrm{~m}$ altitude, whereas the experimental field in Ibaraki prefecture was located in $35^{\circ} .99^{\prime} \mathrm{N}, 140^{\circ} .20^{\prime} \mathrm{E}, 25 \mathrm{~m}$ altitude. Mean temperatures for the cultivation period in Hokkaido and Ibaraki prefecture were $15.4{ }^{\circ} \mathrm{C}$ and $8.3^{\circ} \mathrm{C}$, respectively. Additionally, soil type and soil texture of 2 cultivation locations were differed. The soil type and soil texture in Ibaraki prefecture were andosol and loam, and that in Hokkaido were alluvial soil and sandy loam.

Two-way ANOVA of the $\beta$-eudesmol/hinesol content ratio identified significant differences for the cultivation location in terms of genotypes $(G)$, cultivation locations $(\mathrm{L})$, and $\mathrm{G} \times \mathrm{L}$ interaction; however, mean square for cultivation location and $\mathrm{G} \times \mathrm{L}$ interaction were smaller than that for genotype (Table 3). Additionally, broad-sense heritability of the $\beta$-eudesmol/hinesol content ratio was high at 0.98 in two-way ANOVA of variance components (Table 3). Furthermore, a minimal qualitative interaction was observed between genotype and cultivation location (Fig. 5), and the correlation coefficient for

Table 2 Two-way ANOVA of $\beta$-eudesmol/hinsol content ratio in A. lancea lines grown in 2016 and 2017

\begin{tabular}{lllllc}
\hline & Df & Mean Sq & P-value & $\begin{array}{l}\text { Variance } \\
\text { components }\end{array}$ & $\begin{array}{l}\text { Broad-sense } \\
\text { heritability }\end{array}$ \\
\hline Year $(\mathrm{Y})$ & 1 & 0.41 & $<2 \mathrm{e}-16^{\mathrm{a}}$ & & 1.00 \\
Genotype $(\mathrm{G})$ & 5 & 7.63 & $<2 \mathrm{e}-16^{\mathrm{a}}$ & 0.19 & \\
GXY interaction & 5 & 0.002 & 0.70 & -0.0001 & \\
Residuals & 228 & 0.004 & & 0.004 & \\
\hline
\end{tabular}

Df degree of freedom, Mean Sq Mean square; ${ }^{a} P<0.01$ the $\beta$-eudesmol/hinesol content ratio between the two cultivation locations was 0.97 (Fig. 6).

\section{Discussion}

In this study, $\beta$-eudesmol content was positively correlated with hinesol content in all A. lancea clonal lines (Fig. 1). Takeda et al. [14] similarly showed a strong correlation between $\beta$-eudesmol and hinesol content in $A$. lancea clonal lines. Additionally, variations of the $\beta$ eudesmol/hinesol content ratio within an $A$. lancea clonal line were smaller than those among varietals (Fig. 2), and broad-sense heritability of the $\beta$-eudesmol/ hinesol content ratio was high (Table 1), suggesting strong effects of genetic factors. These data indicate that selective breeding is an effective strategy for stabilizing the $\beta$-eudesmol/hinesol content ratio.

In analyses of the $\beta$-eudesmol/hinesol content ratio, we found no interactions between genotype and cultivation year, and mean square for genotype was higher than that for cultivation year (Table 2). In addition, we demonstrated high broad-sense heritability of the content ratio (Table 2), and a high positive correlation between the two cultivation years (Fig. 4). These results indicate that proportion of genetic variation in total variance is higher than annual variation, and the $\beta$-eudesmol/hinesol content ratio remains stable irrespective of interannual differences in environmental conditions.

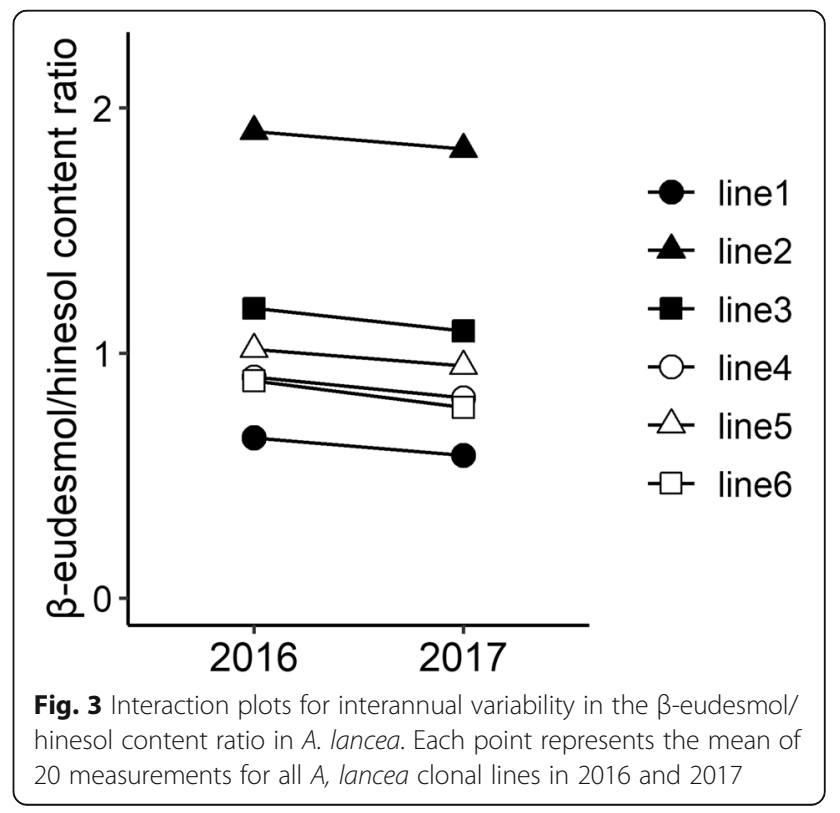




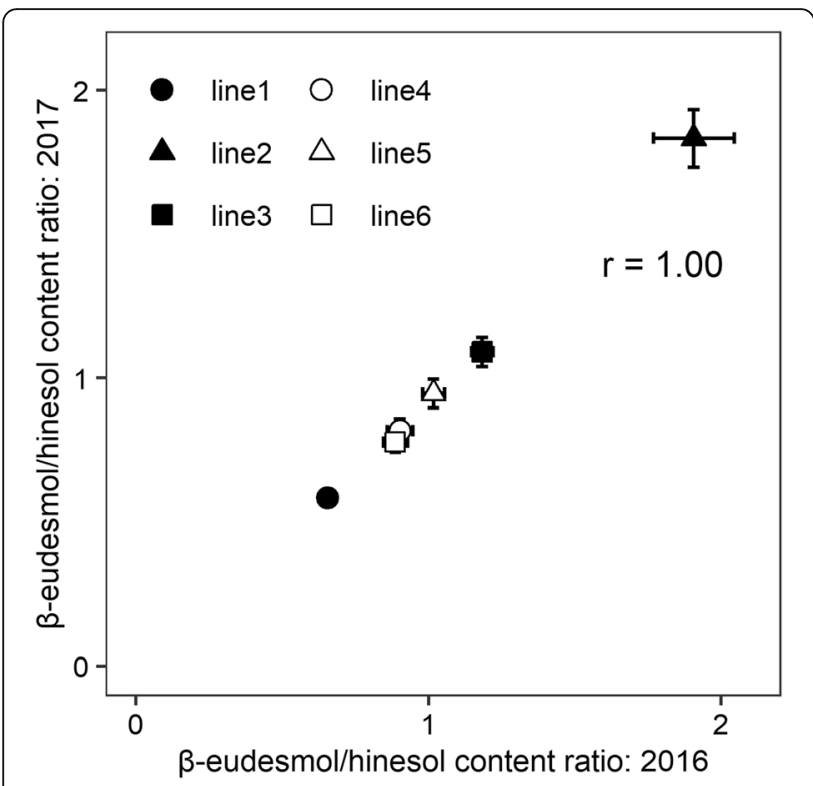

Fig. 4 Correlation of the $\beta$-eudesmol/hinesol content ratio in $A$. lancea clonal lines grown in 2016 and 2017. Each point represents the mean of 20 measurements in 2016 and 2017, whereas bars indicate standard deviations

The two-way ANOVA result for the comparative study of cultivation location identified significant differences in not only genotypes but also cultivation locations and $\mathrm{G} \times$ $\mathrm{L}$ interaction (Table 3). Also, variations of cultivation location and $\mathrm{G} \times \mathrm{L}$ interaction were higher than that of cultivation years and $\mathrm{G} \times \mathrm{Y}$ interaction (Table 3). These results might be caused by variation of environmental conditions such as average temperature and soil conditions [16]. The environmental conditions of two cultivation locations were more varied than that of two cultivation years [16]. However, the broad-sense heritability and correlation coefficient between the two cultivation locations was exhibited high-values (Table 3 and Fig. 6), suggesting that the ratio of genetic variation in total variance is relatively larger than that of the environmental variation (cultivation location). These data indicate that the $\beta$-eudesmol/hinesol content ratio has wide adaptability in A. lancea.

In our previous study of the same samples, we determined the effects of environmental factors on $\beta$ eudesmol and hinesol content [16]. We showed that the

Table 3 Two-way ANOVA of $\beta$-eudesmol/hinsol content ratio in A. lancea lines grown in 2 cultivation locations

\begin{tabular}{lccccc}
\hline & Df & Mean Sq & P-value & $\begin{array}{l}\text { Variance } \\
\text { components }\end{array}$ & $\begin{array}{c}\text { Broad-sense } \\
\text { heritability }\end{array}$ \\
\hline Location $(\mathrm{L})$ & 1 & 1.21 & $<2 \mathrm{e}-16^{\mathrm{a}}$ & & 0.98 \\
Genotype $(\mathrm{G})$ & 5 & 6.44 & $<2 \mathrm{e}-16^{\mathrm{a}}$ & 0.17 & \\
$\mathrm{G} \times \mathrm{L}$ interaction & 5 & 0.12 & $<2 \mathrm{e}-16^{\mathrm{a}}$ & 0.01 & \\
Residuals & 206 & 0.01 & & 0.01 & \\
\hline
\end{tabular}

Df degree of freedom, Mean Sq Mean square; ${ }^{a} P<0.01$

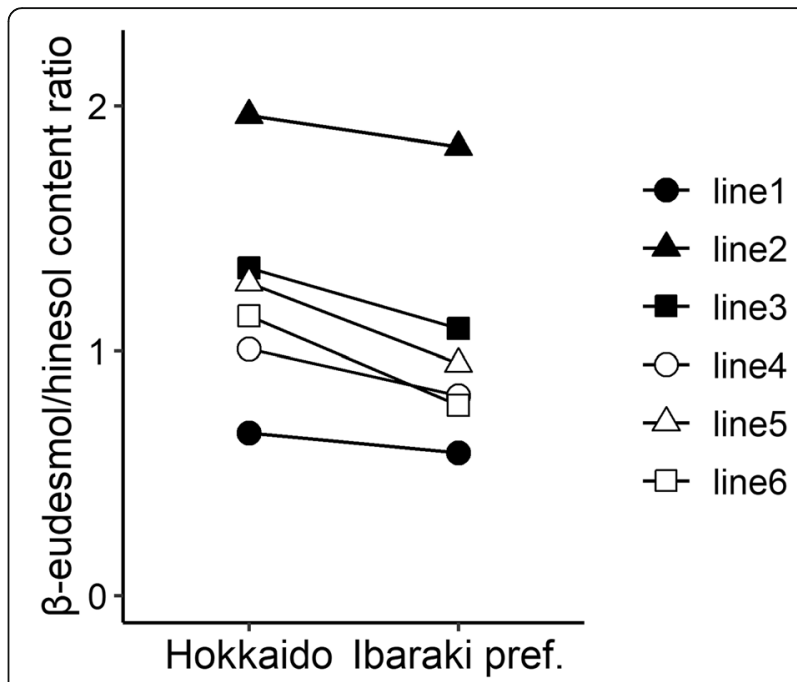

Fig. 5 Interaction plots of $\beta$-eudesmol/hinesol content ratio in $A$. lancea lines grown in 2 cultivation locations. Data are presented as the mean of biological replicates for $A$. lancea clonal lines grown in Hokkaido; lines 1,4 , and $6(n=20)$, line $2(n=12)$, line $3(n=8)$, and line $5(n=18)$. Twenty replicates were generated for each $A$. lancea clonal lines grown in Ibaraki Prefecture

broad-sense heritability of this ratio was higher than that of absolute $\beta$-eudesmol and hinesol content, and that the effects of cultivation year and location on the $\beta$ eudesmol/hinesol content ratio were smaller than on $\beta$ eudesmol and hinesol content [16].

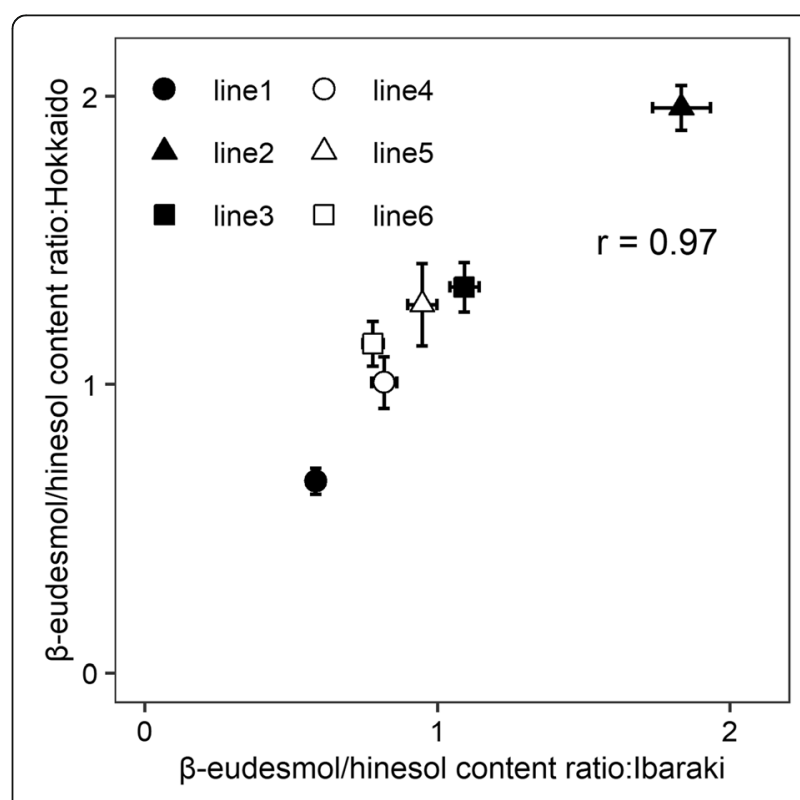

Fig. 6 Correlation of $\beta$-eudesmol/hinesol content ratio in A. lancea lines grown in 2 cultivation locations. Data are presented as mean and standard deviation. The number of biological replicates for all $A$. lancea clonal lines grown in Hokkaido is as follows: lines 1,4 , and $6(n=20)$; line $2(n=12)$; line $3(n=8)$; and line $5(n=18)$. Twenty replicates were generated for each $A$. lancea clonal line grown in Ibaraki Prefecture 
In general, sesquiterpenoids are induced by plant hormones produced in response to biotic and abiotic stresses [17]. In A. lancea, $\beta$-eudesmol and hinesol production is reportedly induced by plant hormones, such as jasmonic acid and absisic acid, through symbiosis with endophytes $[18,19]$. Soil acidity was shown to induce $\beta$-eudesmol accumulation in a previous study on $A$. lancea rhizomes [20]. These investigations show that absolute $\beta$-eudesmol and hinesol content vary with environmental factors. In contrast, we showed limited effects of environmental factors on the $\beta$-eudesmol/hinesol content ratio, corresponding to high broad-sense heritability of this ratio. $\beta$-Eudesmol and hinesol have the same chemical structure and molecular weight; therefore they likely have closely related biosynthetic pathways [5]. Hence, it is possible that the $\beta$-eudesmol/hinesol content ratio is genetically controlled in A. lancea. Recent transcriptome analyses of $A$. lancea identified several candidate genes related to sesquiterpenoid biosynthesis [11, 21]. Additionally, high levels of single- nucleotide polymorphisms have been detected in cDNA libraries of $A$. lancea leaf, stem, and root tissues [22]. These findings highlight the possibility of developing a marker-assisted selection strategy based on the $\beta$-eudesmol/hinesol content ratio using genetic association analysis.

Herein, we indicate that the $\beta$-eudesmol/hinesol content ratio in $A$. lancea rhizomes is highly dependent on genetic factors and suggest that clonal propagation is effective for stabilizing this ratio in A. lancea. A. lancea tissues have been propagated in vitro, particularly tip tissues have been cultured extensively [23]. In particular, Shoyama et al. [24] indicated that 6-benzylaminopurine facilitates shoot propagation, and gibberellin stimulates root enlargement in $A$. lancea. Thus, $A$. lancea strains with a stable $\beta$-eudesmol/ hinesol content ratio could be propagated through mass propagation.

\section{Conclusion}

We demonstrate that the heritability of $\beta$-eudesmol/ hinesol content ratio is high and that the effects of genetic factors were stronger than that of environmental factors such as cultivation location and year. Our findings suggest that selective breeding and clonal propagation are effective strategies for the production of $A$. lancea with stable qualities for use in the production of crude drugs. Further molecular biological analyses such as isplation of genes related to biosynthesis of sesquiterpenoids such as $\beta$-eudesmol and hinesol are warranted.

\section{Methods}

\section{Plant materials}

Twenty-five $A$. lancea clones (lines 1-25) were propagated from rhizomes of a single plant as described previously [16]. In brief, original seed of $A$. lancea were obtained from another previous study [25]. These seeds were originated from China. According to morphological characteristics, the plants were identified as A. lancea by the first author. Voucher specimens (THS33885) were deposited at the herbarium stock room of the Botanical Raw Materials Research Laboratories, Tsumura \& Co. Japan.

\section{Cultivation of $A$. lancea}

Plants were cultivated following the method described in a previous study [16]. To estimate the broad-sense heritability of the $\beta$-eudesmol/hinesol content ratio in A. lancea rhizomes, 25 clonal lines were grown in an experimental field in Ami Town, Inashiki-gun, Ibaraki Prefecture $\left(35^{\circ} .99^{\prime} \mathrm{N}, 140^{\circ} .20^{\prime} \mathrm{E}\right)$, Japan, in 2017. Rhizomes of these $A$. lancea clonal lines were cut into $50-\mathrm{g}$ sections; planted on November 25, 2016; and harvested on November 23, 2017. The number of biological replicate plants for each clonal line was as follows: lines $1-17$ $(n=20)$, lines 18-24 $(n=10)$, line $25(n=5)$.

To evaluate $\mathrm{G} \times \mathrm{E}$ interaction and the effects of environmental factors, 6 clonal lines were grown in different years and in different locations. To examine the effects of cultivation year, 6 clonal lines (lines 1-6) were cultivated in 2016 in an experimental field located in Ibaraki Prefecture. We planted the six clones on November 25, 2015 and harvested them on November 23, 2016. These experiments were performed with 20 biological replicates for each clonal line. In addition, to evaluate the effects of location, six clonal lines were grown in 2017 in another experimental field at Kyowa-Town, Hokkaido $\left(43^{\circ} .01^{\prime} \mathrm{N}, 140^{\circ} .53^{\prime} \mathrm{E}\right)$, Japan. Rhizomes of the 6 clonal lines were divided into $50 \mathrm{~g}$ sections and we cultivated the 6 clonal lines from October 21, 2016, to October 19, 2017, in Hokkaido. This experiment was performed with 8-20 biological replicates for each clonal line as follows: line $1(n=20)$, line $2(n=12)$, line $3(n=8)$, line $4(n=20)$, line $5(n=18)$, and line $6(n=20)$.

\section{Extraction of sesquiterpenoids and gas chromatography- mass spectrometry (GC-MS) analysis}

$\beta$-Eudesmol and hinesol content in A. lancea rhizomes were determined as described previously [16]. The A. lancea rhizomes were dried in a convection drying oven (RY120HG, ALP Co., Ltd., Japan) at $50^{\circ} \mathrm{C}$ for 7 days and pulverized using a vibrating rod mill (TI-200, Cosmic Mechanical Technology, Co. Ltd., Japan) for GC-MS analysis. The powder samples of $A$. lancea rhizomes were accurately weighed at $0.5 \mathrm{~g}$, and extracted with $\mathrm{n}$-hexane $(25 \mathrm{ml})$ using a recipro shacker (SR-1, TAITEC Co., Japan) for $15 \mathrm{~min}$, followed by centrifugation $(1660 \times g, 10 \mathrm{~min})$. After collection of the supernatant, the residues were re-extracted with n-hexane $(20 \mathrm{ml})$ in a same manner. An internal standard (I.S.), phenanthrene $(1.5 \mathrm{mg}, 1 \mathrm{ml}$ in $\mathrm{n}$-hexane), was added to the combined supernatants in $50 \mathrm{ml}$-volumetric flask, and the solutions were made up by adding $\mathrm{n}$-hexane to a 
total volume of $50 \mathrm{ml}$. The analyses were conducted using an Agilent 7890A gas chromatograph (GC) coupled to a 5975C mass spectrometer (MS) (Agilent Technologies, Palo Alto, CA, USA). Sample solutions $(1 \mu \mathrm{L})$ were injected into a DB-WAX capillary column (polyethylene glycol, 30 $\mathrm{m} \times 250 \mu \mathrm{m}$ i.d., $0.25 \mu \mathrm{m}$ film thickness; Agilent J\&W Scientific, Folsom, CA) in a split ratio of 50:1. Helium was used as the carrier gas at a flow rate of $1 \mathrm{~mL} / \mathrm{min}$. The injector temperature was set at $240^{\circ} \mathrm{C}$. The column oven temperature was initially held at $160^{\circ} \mathrm{C}$ for $2 \mathrm{~min}$ after injection, and programmed to increase from 160 to $200^{\circ} \mathrm{C}$ at a rate of $5{ }^{\circ} \mathrm{C} / \mathrm{min}$, then increase from 200 to $240{ }^{\circ} \mathrm{C}$ at a rate of $8{ }^{\circ} \mathrm{C} / \mathrm{min}$, and hold at $240^{\circ} \mathrm{C}$ for $5 \mathrm{~min}$. The interface temperature was set at $240^{\circ} \mathrm{C}$. The MS was operated in an electron impact ionization at $70 \mathrm{eV}$ and the temperatures of the ion source and the quadrupole mass spectrometer were set at $230^{\circ} \mathrm{C}$ and $150^{\circ} \mathrm{C}$, respectively. Total ion current (TIC) chromatograms were acquired in a mass range of 40-500 amu using an Agilent MSD Chemstation software (version E.02.00.493).

The quantitative analyses of $\beta$-eudesmol and hinesol were calculated on the basis of peak-area ratio to the I.S. in TIC chromatogram and regression analyses were performed. Standards of $\beta$-eudesmol (14.08 mg) and hinesol (20.16 mg), provided by Tsumura \& Co. (Japan), were initially dissolved in each $10 \mathrm{~mL}$ hexane, and then stepwisediluted with hexane followed by adding the I.S. $(1.5 \mathrm{mg}$, in $1 \mathrm{~mL}$ hexane) to make series of the standard solutions ranging in concentration from 0.005 to $0.7 \mathrm{mg} / \mathrm{mL}$ of each compounds. The correlation coefficients for the standards of $\beta$-eudesmol and hinesol were 0.99 and 1.00 , respectively. The contents of the sesquiterpenoids were expressed based on the dry weight of the powdered sample.

\section{Statistical analysis}

Statistical analyses were performed using $\mathrm{R}$ (version 3.5.0). To evaluate the effects of genetic factors on the $\beta$ eudesmol/hinesol content ratio, one-way ANOVA was performed using the data of $A$. laneca clonal line grown in an experimental field. Broad-sense heritability $\left(h_{B}\right)$ was calculated from variance components in ANOVA as follows: $h_{B}=\sigma_{G}^{2} /\left(\sigma_{G}^{2}+\sigma_{E}^{2}\right)$, where $\sigma_{G}^{2}$ is genotypic variance and $\sigma_{E}^{2}$ is environmental variance $[26,27]$. Effective numbers of replicates ( $r$ ) for estimating $\sigma^{2}{ }_{G}$ were calculated as follows: $r=\left(\sum_{i=1}^{a} r_{i}-\sum_{i=1}^{a} r_{i}^{2} / \sum_{i=1}^{a} r_{i}\right) /(\mathrm{a}-1)$, where $\mathrm{a}$ is the number of clonal lines [28].

To assess $\mathrm{G} \times \mathrm{E}$ interaction and the effects of environmental factors, we performed analyses of the $\beta$-eudesmol/ hinesol content ratio with two-way ANOVA and Pearson's correlation tests as well as compared A. lancea grown in different years and at different locations. Further, broadsense heritability was estimated from two-way ANOVA values using the following formula: $h_{B}=\sigma_{G}^{2} /\left[\sigma_{G}^{2}+\right.$ $\left(\sigma_{G \times E}^{2} / \mathrm{e}\right)+\left(\sigma_{E}^{2} /\right.$ re $\left.)\right]$, where $\sigma_{G \times E}^{2}$ is the $G \times E$ interaction variance and $\mathrm{e}$ is the number of experiments (cultivation year or location) [29].

\section{Abbreviations}

ANOVA: Analysis of variance; E: Environment; G×E: Genotype-environment interaction; G: Genotype; GC/MS: Gas chromatography/mass spectrometry; $h_{B}$ : Broad-sense heritability; L: Cultivation location; Y: Cultivation year

\section{Acknowledgements}

We are grateful to Assistant Prof. Yosuke Yoshioka (University of Tsukuba) for technical support in data analysis. We thank Mr. Mikio Sakai, Ms. Akiko Uetake, and Ms. Terue Kurosawa for their assistance with cultivation. We also thank Dr. Kazunori Hashimoto, Dr. Kenji Kondo, and Dr. Yoichiro Nakai for their helpful suggestions.

\section{Authors' contributions}

$\Pi T$ and $\mathrm{BM}$ performed the experiments, $\Pi$ analyzed the data, and wrote the manuscript; RO and HE designed the experiments; and all authors approved the manuscript. All authors read and approved the manuscript.

\section{Funding}

This study was funded by Tsumura \& Co. (https://www.tsumura.co.jp/english/ ). The funder provided support in the form of a salary for $\Pi$ (First author) and BM (Second author). HE (Last author) and RO (Third author) are supervisors at the University of Tsukuba, to which $\Pi$ T belongs, and there is no funding from Tsumura \& Co. The specific roles of these authors have been articulated in the "author contributions" section. Tsumura \& Co. was responsible for the study design, data collection and analysis, decision to publish, and preparation of the manuscript.

Availability of data and materials

The datasets supporting the conclusions and methods description are included within the article.

Ethics approval and consent to participate

Not applicable.

Consent for publication

Not applicable.

\section{Competing interests}

The authors declare that they have no competing interests.

\section{Author details}

'Graduate School of Life and Environmental Sciences, University of Tsukuba, Tsukuba, Ibaraki, Japan. ${ }^{2}$ Botanical Raw Materials Production Department 2, Tsumura \& Co., Ami Town, Ibaraki, Japan. ${ }^{3}$ Botanical Raw Materials Research Laboratories, Tsumura \& Co., 3586 Yoshiwara, Ami-machi, Ibashiki-gun, Ami Town, Ibaraki, Japan. ${ }^{4}$ Faculty of Life and Environmental Sciences, University of Tsukuba, Ten-nodai 1-1-1, Tsukuba, Ibaraki 305-8572, Japan.

Received: 11 November 2019 Accepted: 2 March 2020

Published online: 11 March 2020

\section{References}

1. Shiba M, Kondo K, Miki E, Yamaji H, Morota T, Terabayashi S, Takeda S, Sasaki H, Miyamoto K, Aburada M. Identification of medicinal Atractylodes based on ITS sequences of nrDNA. Biol Pharm Bull. 2006;29:315-20.

2. Kubo M, Nogami M, Nishimura M, Moriura T, Arichi S. Studies of crude drugs about its origin, process and quality. I. the preventive effects of chinese crude drug "Zhu" on experimental stomach ulcer and its pharmacological evaluation (1). Yakugaku Zasshi. 1983;103:442-8.

3. Wang HX, Liu CM, Liu Q, Gao K. Three types of sesquiterpenes from rhizomes of Atractylodes lancea. Phytochemistry. 2008;69:2088-94.

4. Koonrungsesomboon N, Na-Bangchang K, Karbwang J. Therapeutic potential and pharmacological activities of Atractylodes lancea (Thunb.) DC. Asian Pac. J Trop Med. 2014;7:421-8. 
5. Yosioka I, Takahashi S, Hikino H, Sasaki Y. Studies on the constituents of Atractylodes. III. Separation of atractylol into eudesmol and hinesol. Chem Pharm Bull. 1958;7:319-23.

6. Nogami M, Moriura T, Kubo M, Tani T. Studies on the origin, processing and quality of crude drugs. II. Pharmacological evaluation of the Chinese crude drug "Zhu" in experimental stomach ulcer. (2). Inhibitory effect of extract of Atractylodes lancea on gastric secretion. Chem Pharm Bull. 1986;34:3854-60.

7. Satoh K, Nagai F, Ushiyama K, Yasuda I, Akiyama K, Kano I. Inhibition of $\mathrm{Na}^{+}, \mathrm{K}^{+}$-ATPase activity by $\beta$-eudesmol, a major component of atractylodis lanceae rhizoma, due to the interaction with enzyme in the $\mathrm{Na} \cdot \mathrm{E}_{1}$ state. Biochem Pharmacol. 1992:44:373-8.

8. Satoh $\mathrm{K}$, Nagai $\mathrm{F}$, Kano I. Inhibition of $\mathrm{H}^{+}, \mathrm{K}^{+}$-ATPase by hinesol, a major component of So-jutsu, by interaction with enzyme in the E1 state. Biochem Pharmacol. 2000;59:881-6.

9. Masuda Y, Kadokura T, Ishii M, Takeda K, Kitajima J. Hinesol, a compound isolated from the essential oils of Atractylodes lancea rhizome, inhibits cell growth and induces apoptosis in human leukemia HL-60 cells. J Nat Med. 2015;69:332-9.

10. Zhang W, Ouyang Z, Zhao M, Wei Y, Peng H, Wang Q, Guo L. The influences of inorganic elements in soil on the development of famous region Atractylodes lancea (Thunb.) DC. Pharmacogn Mag. 2015;42:337-44.

11. Chen F, Wei YX, Zhang JM, Sang XM, Dai CC. Transcriptomics analysis investigates sesquiterpenoids accumulation pattern in different tissues of Atractylodes lancea (Thunb.) DC. Plantlet. Plant Cell Tissue Organ Cult. 2017; 130:73-90.

12. Takeda O, Miki E, Higuchi M, Okada M. Historical investigation on quality evaluation of Atractylodes lancea rhizome (Cangzhu; Soujutsu) (II): on the Japanese herbal literature. Jpn J Hist Pharm. 1998:33:24-8.

13. Takeda O, Miki E, Terabayashi S, Okada M, Lu Y, He SA. Variation of essential oil components of Atractylodes lancea growing in China. Natural Med. 1995; 49:18-23.

14. Takeda O, Miki E, Terabayashi S, Okada M, He SA, Sashida Y. Seasonal variation of essential oil components in Atractylodes lancea (THUNB.) DC. Propagated by division of their rhizomes. Chem Pharm Bull. 1996:44:823-8.

15. Zhen $O$, Zhang L, Zhao M, Wang P, Wei Y, Fang J. Identification and quantification of sesquiterpenes and polyacetylenes in Atractylodes lancea from various geographical origins using GC-MS analysis. Braz J Pharmacogn. 2012:22:957-63.

16. Tsusaka T, Makino B, Ohsawa R, Ezura H. Genetic and environmental factors influencing the contents of essential oil compounds in Atractylodes lancea. PLoS One. 2019;14:e0217522.

17. Sudha G, Ravishankar GA. Involvement and interaction of various signaling compounds on the plant metabolic events during defense response, resistance to stress factors, formation of secondary metabolites and their molecular aspects. Plant Cell Tissue Organ Cult. 2002;71:181-212.

18. Ren CG, Dai CC. Jasmonic acid is involved in the signaling pathway for fungal endophyte-induced volatile oil accumulation of Atractylodes lancea plantlets. BMC Plant Biol. 2012;12:128

19. Wang XM, Yang B, Ren CG, Wang HW, Wang JY, Dai CC. Involvement of abscisic acid and salicylic acid in signal cascade regulating bacterial endophyte-induced volatile oil biosynthesis in plantlets of Atractylodes lancea. Physiol Plant. 2015;153:30-42.

20. Yuan Y, Liu YJ, Huang LQ, Cui GH, Fu GF. Soil acidity elevates some phytohormone and $\beta$-eudesmol contents in roots of Atractylodes lancea. Russ J Plant Physiol. 2009;56:133-7.

21. Huang Q, Huang X, Deng J, Liu H, Liu Y, Yu K, Huang B. Differential gene expression between leaf and rhizome in Atractylodes lancea: a comparative transcriptome analysis. Front Plant Sci. 2016;7:1-13.

22. Ahmed S, Zhan C, Yang Y, Wang X, Yang T, Zhao Z, Zhang Q, Li X. The transcript profile of a traditional Chinese medicine, Atractylodes lancea, revealing its sesquiterpenoid biosynthesis of the major active components. PLoS One. 2016;11:e80151975.

23. Hiraoka N, Yamada N, Kodama T, Tomita Y. In vitro propagation of Atractylodes lancea. Plant Cell Rep. 1984;3:85-7.

24. Shoyama Y, Tareno R, Nishioka I. Clonal maultiplication of Atractylodes lancea by tip tissue culture. Shoyakugaku Zasshi. 1987:41:313-7.

25. Takeda O, Miki E, Terabayashi S, Okada M, Lu Y, He HS, He SA. A comparative study on essential oil components of wild and cultivated Atractylodes lancea and A. chinensis. Planta Med. 1996;62:444-9.

26. Ukai Y. Genetic analysis of quantitative traits. Original ed. Tokyo: IgakuShuppan Inc.; 2002.
27. Burton GW, Devane EH. Estimating heritability in tall fescue (Festuca arundinacea) from replicated clonal material. Agron J. 1953;45:478-81.

28. Yoshida M. Design of experiments for animal husbandry. 8th ed. Tokyo: Yokendo co. Ltd.; 1998.

29. Toker C. Estimates of broad-sense heritability for seed yield and yield criteria in faba bean (Vicia faba L.). Hereditas. 2004;140:222-5.

\section{Publisher's Note}

Springer Nature remains neutral with regard to jurisdictional claims in published maps and institutional affiliations.

\section{Ready to submit your research? Choose BMC and benefit from:}

- fast, convenient online submission

- thorough peer review by experienced researchers in your field

- rapid publication on acceptance

- support for research data, including large and complex data types

- gold Open Access which fosters wider collaboration and increased citations

- maximum visibility for your research: over $100 \mathrm{M}$ website views per year

At $\mathrm{BMC}$, research is always in progress.

Learn more biomedcentral.com/submissions 